\title{
SUBCUTANEOUS TISSUE RESPONSES IN RATS TO INJECTION OF FINE PARTICLES OF SYNTHETIC HYDROXYAPATITE CERAMIC
}

\author{
SHIN-ICHI SHIMIZU \\ Department of Oral Pathology, Niigata University School of Dentistry, Gakkocho Dori, Niigata 951, Japan
}

\begin{abstract}
To clarify tissue responses to hydroxyapatite ceramic (HAP), time course observation of lesions induced by subcutaneous injections of fine particles of HAP was performed by light and electron microscopy: The description was based on the division of the particles into smaller-sized and larger-sized ones. Smaller particles, ranging in diameter from 0.65 to a few micra and scattered in the tissue, were phagocytized by macrophages in extremely early stages. Phagocytized particles formed phagosomes and phago-lysosomes containing one or more particles. In later stages, macrophages laden with smaller particles were widely distributed and occasionally observed in perivascular areas. Some of the phagocytized particles were resolved in phago-lysosomes. In contrast, larger particles measuring several micra in diameter were not phagocytized, being instead surrounded by numerous macrophages and multinucleated giant cells. Infiltrating macrophages at the injection sites gathered densely, closely approximating one another by the interdigitation of numerous microprojections in a zipper-like manner. The formation of a fibrous capsule was not evident, though the fibroblasts were gradually increased in number in and around the lesions. These results suggest that HAP particles are recognized as foreign bodies and provoke foreign body reactions in cells, principally that in mononuclear phagocytes and their derivates.
\end{abstract}

Synthetic hydroxyapatite ceramic (HAP) has been recently developed as a biomaterial, and has been spotlighted for its excellent qualities of biocompatibility and osteo-conduction when employed in bone tissue $(7,14,15)$. In dentistry, HAP is frequently employed in dental implants and the reconstruction of bone defects or the augmentation of bone necessitated by various causes such as the resection of tumors or periodontal diseases.

As to soft tissue responses to large particles of HAP which could not be phagocytized by macrophages, inflammatory reactions resolved immediately after implantation and the particles were encapsulated by fibrovascular or fibrous tissue intermingling a few macrophages and multinucleated giant cells. However, these tissue responses were interpreted as indicating that HAP had good biocompatibility without any tissue damage $(4,7,12)$.

Biomaterials have indispensable qualities, varying according to site and usage, such as durability, non-toxicity and biocompatibility. The first two were almost satisfactorily realized. The third remains under consideration from the standpoint of the encapsulation of applied biomaterials by fibrous tissue, among others. In clinical applications of biomaterials, 
special attention toward the tissue responses especially biocompatibility of these materials must be given.

The present study aims to elucidate the soft tissue response produced by subcutaneous administration of fine particles of HAP with three different diameters, as have been reported by several authors $(13,22)$.

\section{MATERIALS AND METHODS}

Twenty-one normal, healthy, male rats of the Wistar strain weighing from 120 to $150 \mathrm{~g}$ were used. The particles of HAP $\left[\mathrm{Ca}_{10}\left(\mathrm{PO}_{4}\right)_{6}(\mathrm{OH})_{2}\right]$ used in this series of experiments were 0.65 , $2-10,10-15 \mu \mathrm{m}$ in diameter and sintered at $1,050^{\circ} \mathrm{C}$ for $4 \mathrm{~h}$. The suspensions containing these particles, the proportion being $0.02 \mathrm{~g} / \mathrm{ml}$ in physiological solution, were sterilized in autoclave. Each rat was shaved of its abdominal hair, disinfected by ethanol and then injected with $0.1 \mathrm{ml}$ of these suspensions, respectively, into the abdominal subcutaneum at six sites. The injections were done with tuberculin syringes with no. 25 needles. The rats were then sacrificed on the 1 st, 3 rd, 5 th, 7th, 14th, 21st and 28th days after injection. For the control, only physiological solution was injected in the same manner. These experiments were performed three times to confirm reproducible results.

For light microscopy, resected tissues from each rat were fixed in 10\% buffered formalin. Some of them were then decalcified in $5 \%$ ethylendiaminẹtetraacetic acid (EDTA, pH 7.4). The decalcified and undecalcified tissues were embedded in paraffin, and thin sections were stained with hematoxylin and eosin, Azan Mallory, Elastica van Gieson staining, the silver impregnation method and periodic. acid-Schiff (PAS) reaction. Stable form acid phosphatase (SAPase) was stained according to Eggerts et al. (5). Spread preparations of normal, non-irritated subcutaneous connective tissue stained with Giemsa solution were also examined.

For electron microscopy, resected tissues and buffy coats of peripheral blood from each rat were immediately fixed in $2.5 \%$ glutaraldehyde in $0.1 \mathrm{M}$ cacodylate buffer, $\mathrm{pH} 7.4$ at $4^{\circ} \mathrm{C}$ for $6 \mathrm{~h}$. In the fixative, the tissues were divided into small pieces. Some of them were decalcified in 5\% EDTA. The small pieces of the tis- sues and buffy coats were postfixed in $1 \%$ osmium tetroxide for $2 \mathrm{~h}$.

For ruthenium red staining, small pieces of resected tissues were fixed in $2.5 \%$ glutaraldehyde in $0.1 \mathrm{M}$ cacodylate buffer containing $500 \mathrm{ppm}$ ruthenium red. They were then decalcified in 5\% EDTA and postfixed in $1 \%$ osmium tetroxide containing $500 \mathrm{ppm}$ ruthenium red (11).

For peroxidatic reaction, small pieces of resected tissues and buffy coats were fixed in $1.25 \%$ glutaraldehyde in cacodylate buffer, $\mathrm{pH}$ 7.4 , at $4^{\circ} \mathrm{C}$ for $30 \mathrm{~min}$ and rinsed in the same buffer. They were incubated with $0.05 \%$ diaminobenzidine medium containing $0.02 \%$ hydrogen peroxide for $2 \mathrm{~h}$ (6). They were decalcified in 5\% EDTA and postfixed in $1 \%$ osmium tetroxide.

All specimens mentioned above were dehydrated in a series of graded ethanols and embedded in Epon 812. Ultra-thin sections were stained doubly with uranyl acetate and lead citrate or uranyl acetate alone, and examined under a Hitachi H-300 electron microscope.

\section{RESULTS \\ Blood Monocytes}

Under the electron microscope, blood monocytes were round to oval in shape with a few cytoplasmic projections. The relatively narrow cytoplasm contained a few mitochondria, Golgi apparatuses and poorly developed rough endoplasmic reticula. Characteristically, lysosomal granules showing a variety of forms, sizes and numbers were distributed in the cytoplasm. The nuclei were oval to beanshaped and heterochromatin masses were adherent to the nuclear envelope.

\section{Normal, Non-irritated Subcutaneous Connective Tissue}

A small number of tissue macrophages, and a large number of fibroblasts and mast cells were dispersed in entwining collagen fibers in spread preparations. In paraffin sections, the few tissue macrophages possessed relatively ample basophilic cytoplasm with oval or beanshaped nuclei. They were strongly positive in PAS reaction and faintly stained for SAPase. Fibroblasts possessed oval to spindle-shaped 


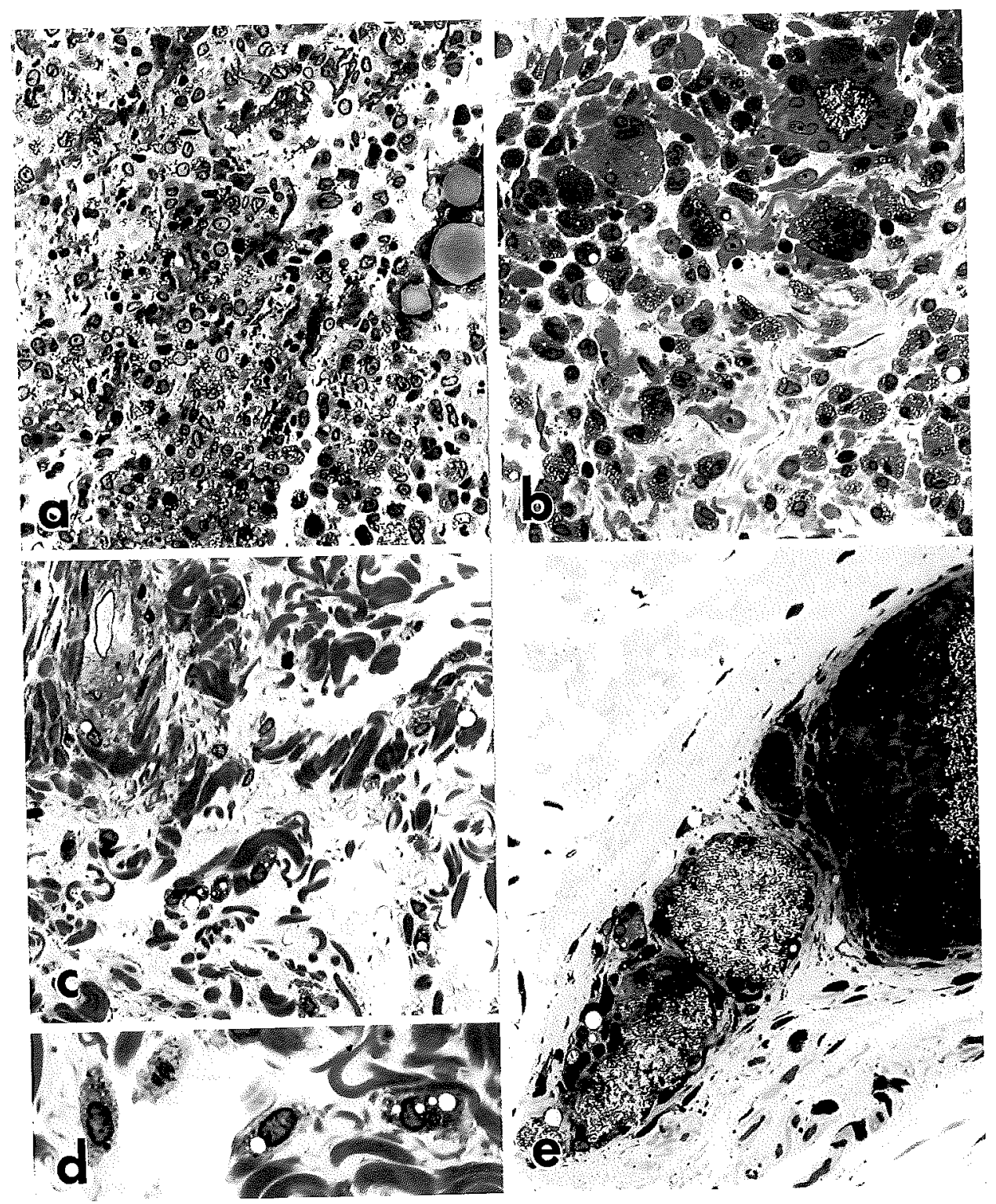

Fig. 1 Light micrographs of the lesions at various stages (group-A). a: Numerous infiltrating macrophages phagocytize smaller particles. The particles are still present in extracellular spaces (1st day). b: Enlarged macrophages and a few multinucleated giant cells phagocytize numerous particles (5th day). c: Vacuolated cells are widely distributed (14th day). d: High magnification of vacuolated cells. e: Conglomerations of smaller particles are surrounded by compactly aggregated macrophages, multinucleated giant cells and spindle-shaped cells (28th day). Toluidine blue $a, b$, c, e: $\times 370, \mathrm{~d}: \times 740$

nuclei and pale cytoplasm.

Under the electron microscope, tissue macrophages showed oval to bean-shaped nuclei, occasionally with irregular indenta- tions and heterochromatin gathered at the periphery in various degrees. The cell surfaces with numerous cytoplasmic microprojections were covered with amorphous or fine granular 
materials, or glycocalyx. The abundant cytoplasm contained a small number of mitochondria, Golgi apparatuses, short strands of rough endoplasmic reticula and occasional autophagic vacuoles. Various numbers, sizes and forms of lysosomal granules were characteristic features. Many vacuoles and coated vesicles were present at the periphery of cytoplasm. On the other hand, fibroblasts were spindle-shaped and characterized by numerous well-developed rough endoplasmic reticula.

\section{Histopathological Evaluations of Tissue Responses to HAP}

\section{Gross Findings}

During the course of the experiments, all rats remained healthy, and no unfavorable findings such as discoloration, redness or ulceration were recognized at the injection sites. Injected particles were studded in relatively narrow areas in the subcutaneous connective tissue.

\section{Controls (Subcutaneous Injections of a Physiological Solution)}

On the 1st day, the injection sites appeared slightly edematous. A small number of polymorphonuclear neutrophilic granulocytes (neutrophils) and many, small immature macrophages infiltrated into the injection sites. By the 3rd day, the infiltrations of neutrophils and macrophages had gradually resolved. By the 5th day, inflammatory cells were few or completely absent. The degeneration or necrosis of any cell was almost never observed at the injection sites and surrounding tissues.

\section{Subcutaneous Injections of HAP Particles}

HAP particles of smaller diameter $(0.65 \mu \mathrm{m}$, group-A) were phagocytized by macrophages and multinucleated giant cells. In contrast, larger particles (10-15 $\mu \mathrm{m}$, group-C) were not phagocytized but only surrounded by these cells. In the case of particles of an intermediate size $(2-10 \mu \mathrm{m}$, group-B), the above-mentioned two processes were observed simultaneously. For the convenience of description, the tissue responses were divided into two parts; one for the smaller particles and the other for the larger particles.

\section{i. Tissue responses to the smaller particles}

Light microscopic findings On the 1st day, numerous inflammatory cells infiltrated at the injection sites, dispersing throughout the surrounding tissues. Most of these cells were small and immature macrophages which had oval to bean-shaped nuclei and PAS- and SAPase-positive granules in the cytoplasm. A small number of neutrophils were present. Many of the injected particles were already phagocytized by macrophages; the remainder were still present in the relatively edematous connective tissue (Fig. 1a).

By the 3rd day, infiltrating macrophages had increased in both number and size and gathered at the injection sites. Many small, oval-shaped macrophages were still present in the perivascular areas. Neutrophils were slowly decreased in number. Most of the particles were phagocytized by macrophages and a few multinucleated giant cells.

From the 5 th to 7 th day, edema and neutrophils gradually disappeared to where they could be hardly found at the injection sites. By this time, multinucleated giant cells were often recognized. Nearly all of the particles were phagocytized by macrophages and multinucleated giant cells. The phagocytized particles were thus steadily increased in number (Fig. 1b). They became loosely or compactly aggregated. A poorly defined cellular zone consisting of many macrophages and a few multinucleated giant cells was formed around conglomerations of the particles.

From the 14th to the 28th day, the macrophages and multinucleated giant cells containing the particles became widely but sparsely distributed without evident vascular, fibroblastic or fibrous responses. The largest number of these macrophages was found in perivascular areas. Some enlarged macrophages showed vacuolated appearances (Fig. 1, $\mathrm{c}$ and d). Around the conglomerations of the particles, a cellular zone consisting of macrophages, multinucleated giant cells and spindleshaped cells became thinner and more concentrated. Spindle-shaped cells were slightly increased in number and were present lamellately around the lesions (Fig. 1e).

Electron microscopic findings In early stages, two populations of macrophages were observed at the injection sites (Fig. 2). Imma- 




Fig. 2 Electron micrograph of early stage of the lesion (group-A, 3rd day). The infiltrating macrophages showing various sizes and shapes: only a few smaller particles (arrows) are phagocytized by mature macrophage appearing to be tissue macrophage. $\times 1,700$

ture macrophages resembling blood monocytes were round to oval in shape with blunt cytoplasmic projections. The ovoid nuclei contained dense heterochromatin, and the nucleoli were inconspicuous. Intracytoplasmic organelles were poorly developed and only a few lysosomal granules, rough endoplasmic reticula and mitochondria were observed. Mature macrophages, appearing to be tissue macrophages, were also present, though few in number. These cells were larger than the immature macrophages and were stellate-shaped with numerous microprojections. Intracytoplasmic organelles were well developed. Relatively large numbers of lysosomal granules, small numbers of single strands of rough endoplasmic reticula, a few Golgi apparatuses, many vacuoles and coated vesicles were found in the ample cytoplasm. Nuclei were bean-shaped with irregular indentations, and heterochromatin granules were rather scanty. Subplasmalemmal linear densities, electron dense intracytoplasmic structures found immediately beneath the cell membrane (9), were occasionally observed. These structures were not recognized in blood monocytes nor in immature macrophages. Immature macrophages sequentially differentiated into mature forms of macrophages. Finally, mature form of macrophages and tissue macrophages could not be distinguished from each other on morphological grounds with the passage of time.

On the 1st day, a few particles had already been phagocytized by mature macrophages appearing to be tissue macrophages. The 




Fig. 3 In initial stage of phagocytosis, invagination of the cell membrane and the surrounding of the small particles are seen. Dense aggregations of microfilaments subjacent to the cell membrane, subplasmalemmal microfilaments are present (group-A). $\times 63,000$

phagocytized particles were enclosed by a limiting membrane, forming phagosomes, but were not yet fused with lysosomal granules. Some of the particles lay in connective tissue and were captured by elongated pseudopodia. Immature macrophages did not phagocytize the particles.

From the 3rd to 5 th day, infiltrating immature macrophages showed gradual differentiations in various degrees. Relatively differentiated macrophages began to participate in the phagocytizing process of the particles, and various stages of the process were observed. Firm contacts between particles and cell membranes and the infolding of particles by elongated microprojections with occasional condensation of fine filaments, or subplasmalemmal microfilaments (19), were observed (Fig. 3). Phagocytized particles were steadily increased in number under various conditions in the cytoplasm. Some of phagosomes fused or were fusing with lysosomal granules and formed phago-lysosomes (Fig. 4a). In perivascular areas, some immature macrophages were still present.

By the 7th day, most of the infiltrating immature macrophages had differentiated into the mature form of macrophages. Phagosomes and phago-lysosomes were increased in number. Phago-lysosomes gradually enlarged as the number of particles in them also increased. Around conglomerations of the particles, macrophages lined up in stratified arrangements intermingling a few multinucleated giant cells. These cells became closely packed with one another by intertwining sinuous microprojections. Macrophages in the innermost layer of the cellular zone intruded pseudopodia into interstrices among the particles and phagocytized some particles. A subplasmalemmal linear density was also recognized in multinucleated giant cells. Immature macrophages could be seen at this time.

From the 14th to 28 th day, particle-laden 


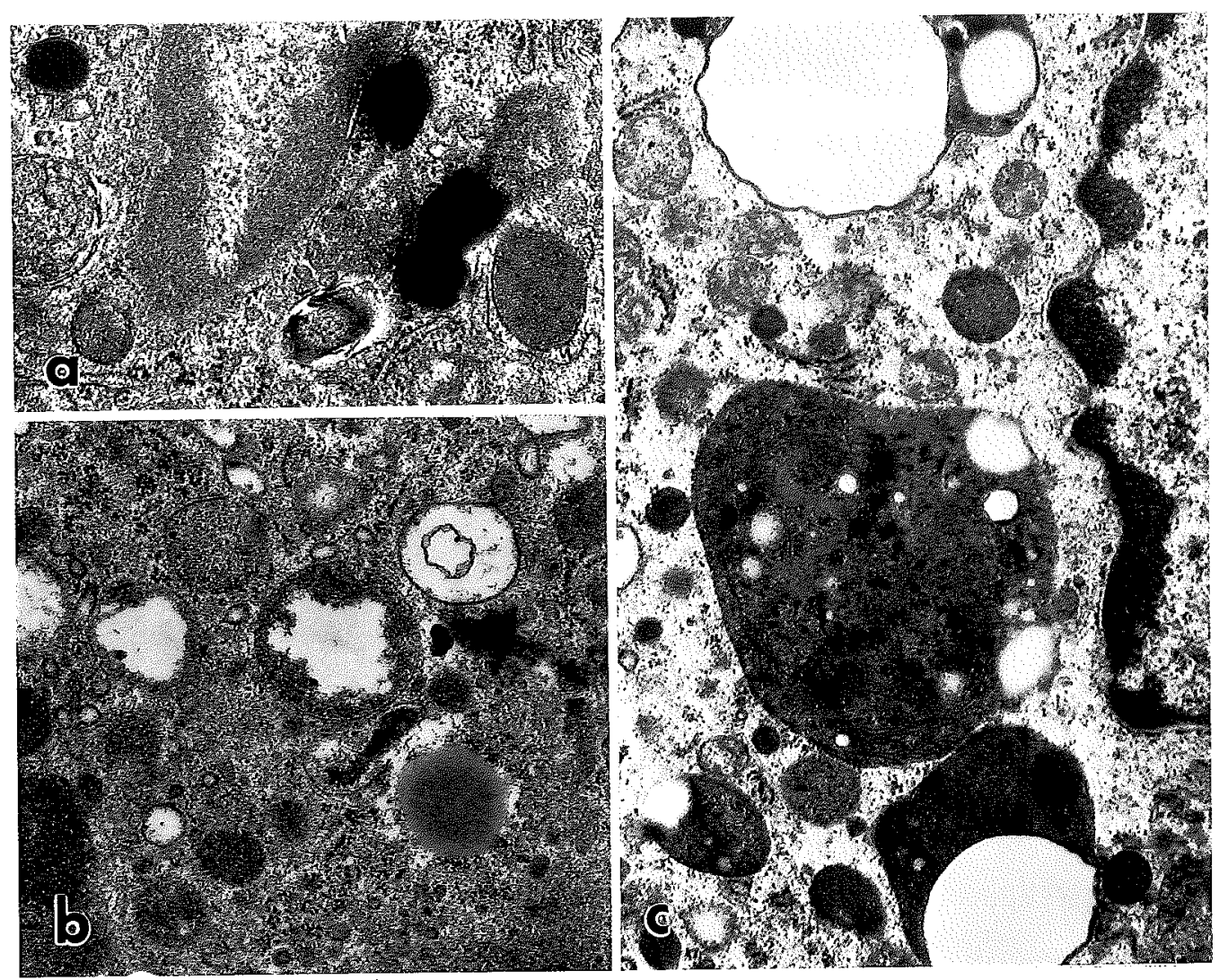

Fig. 4 The processes of intracytoplasmic resolution of phagocytized particles by macrophages (group-A). a: Phagosomes containing particles with or without fusing lysosomal granules. b: Irregular shaped vacuoles and myelin-like lamellar material are seen in phago-lysosomes. c: Large phago-lysosomes are divided into small compartments by membranes and contain variously sized vacuoles. a: $\times 14,000 ; b: \times 22,000 ; c$ : $\times 14,000$

macrophages and multinucleated giant cells became widely dispersed. The above-mentioned vacuolated cells in the light microscopic findings turned out to be not degenerated cells but macrophages containing a few irregular-shaped vacuoles and myelin-like materials in phago-lysosomes (Fig. 4b). Phago-lysosomes containing irregular or round-shaped vacuoles were gradually increased in number and size. Larger ones were composed of double-layered membrane bound small compartments. They were heterogenous in electron density showing regional differences within the phago-lysosome (Fig. 4c). Short fragments of the particles were occasionally recognized in phagolysosomes. Non-resolved particles were also present in the small or large phago-lysosomes. Macrophages containing the particles were gradually increased in number in perivascular areas with time, and were occasionally observed in close proximity to blood vessel walls, and at rare times within them (Fig. 5). Lymphocytes and plasma cells were infrequently observed in the lesions. However, an intimate connection between a macrophage containing particles and a lymphocyte could occasionally be seen. In the cytoplasm of the macrophages, especially those facing lymphocytes, numerous coated vesicles were seen (Fig. 6, a and b). Around conglomerations of the particles, macrophages and multinucleated giant cells closely approximated one another by interdigitating with prominent microprojections, leaving no discernible intercellular spaces. In the periphery of the lesions, spindle-shaped macrophages and fibroblasts were observed. 


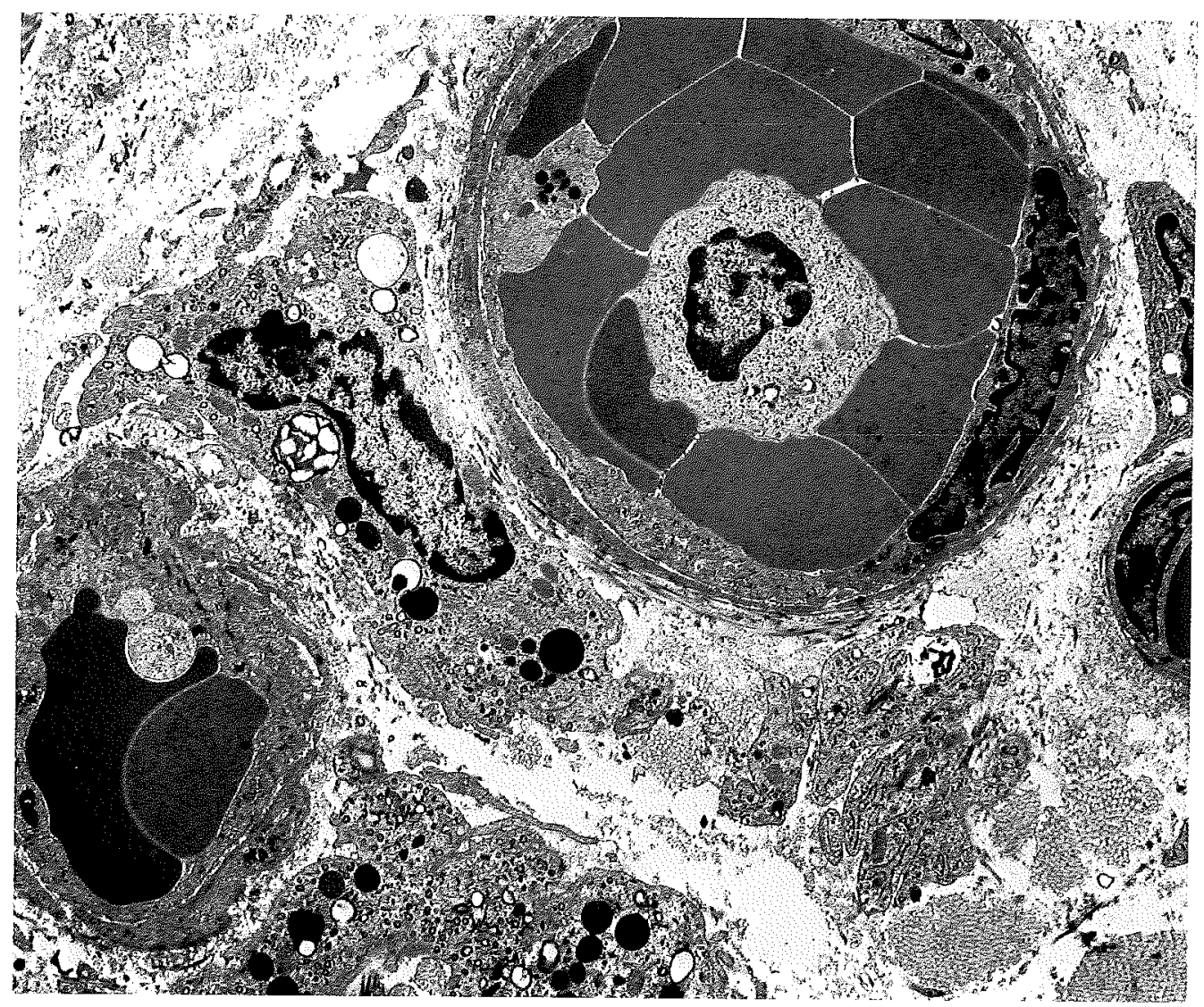

Fig. 5 Smaller particles laden macrophages are often observed in perivascular areas, but rarely in blood vessels (group-B, 28th day). $\times 5,100$. Decalcified

\section{ii. Tissue responses to the larger particles}

Light microscopic findings On the 1st day, numerous immature macrophages infiltrated into relatively edematous injection sites. Some of them already made close contact with larger particles. However, many of them were without any particular connections to the particles (Fig. 7a). A mild acute inflammation was also present.

From the 3rd to 5 th day, infiltrating immature macrophages became gradually enlarged and more densely populated around the particles. Only a few multinucleated giant cells could be encountered. Apart from these lesions, solitarily existing larger particles were completely enclosed by mononucleated or binucleated cells. Small and oval-shaped immature macrophages were still present especially in perivascular areas. Edema and the number of neutrophils decreased by slow degrees.

On the 7th day, the lesions were composed of macrophages and a few multinucleated giant cells and had become relatively well-outlined. They were closely packed against one another and arranged in sheets around the particles.

From the 14 th to 28 th day, the lesions became more compact and restricted. Macrophages and multinucleated giant cells closely arranged themselves around the particles; their cytoplasmic borders were indistinct. In the periphery of the lesions and surrounding tissues, spindle-shaped cells were gradually increased in number. Only a few capillaries and collagen fibers were encountered in the lesion (Fig. 7b). 

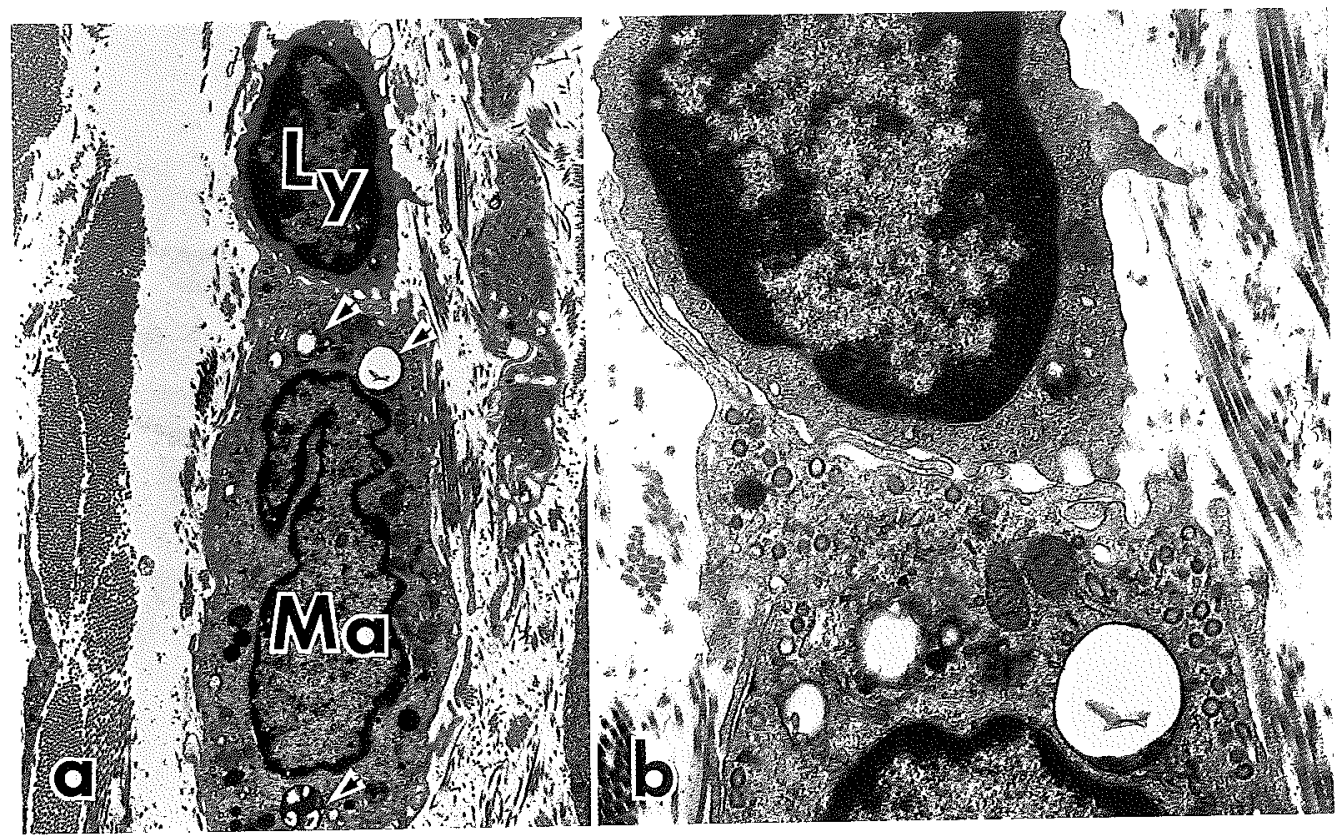

Fig. 6 a: Intimate contact of a macrophage (Ma) containing smaller particles and a lymphocyte (Ly) (group-B, 21st day). Arrowheads indicate decalcified smaller particles. b: Note the many coated vesicles in the cytoplasm of the macrophage facing the lymphocyte. a: $\times 3,500 ; \mathrm{b}: \times 5,500$. Decalcified

SAPase activity was demonstrated in inf1trating macrophages and multinucleated giant cells. In early stages, diffusely infiltrating immature macrophages showed a weak positive reaction in the cytoplasm. In the injection sites, compactly aggregated macrophages and multinucleated giant cells which phagocytized or surrounded particles also showed a positive reaction. However, variations in the intensity of staining reaction in these cells were recognized.

Electron microscopic findings On the 1st day, numerous infiltrating immature macrophages resembling blood monocytes, a few mature macrophages appearing to be tissue macrophages and neutrophils were present in the relatively edematous injection sites. At this time, some of the cells already adhering in various degree to the particles but did not enclose them completely.

On the 3rd day, one or more macrophages lay in close adherence to the particles or enclosed them with an elongated cytoplasm. A small amount of collagen or elastic fibers was still present among the infiltrating cells and between the cell and particles. Infiltrating immature macrophages showed gradual differentiations in various degrees.

By the 5th day, the lesions gradually maturated with new recruitments of a small number of immature macrophages. Injected particles were surrounded by numerous macrophages intermingling a few multinucleated giant cells. These cells began to interdigitate with each other with their elongated microprojections, though narrow intercellular spaces were still present among adjacent cells. Multinucleated giant cells were rich in lysosomal granules, mitochondria and rough endoplasmic reticula. However, irregular cytoplasmic processes and ruffled borders characteristic of osteoclasts, were not observed on their surfaces façing the particles. Solitary larger particles were completely enclosed by macrophages (Fig. 8a). Subplasmalemmal linear densities and subplasmalemmal microfilaments were occasionally found in relatively differentiated macrophages (Fig. 8b).

By the 7 th day, mature macrophages and multinucleated giant cells closely approximat ed each other by exchanging many tentacular 


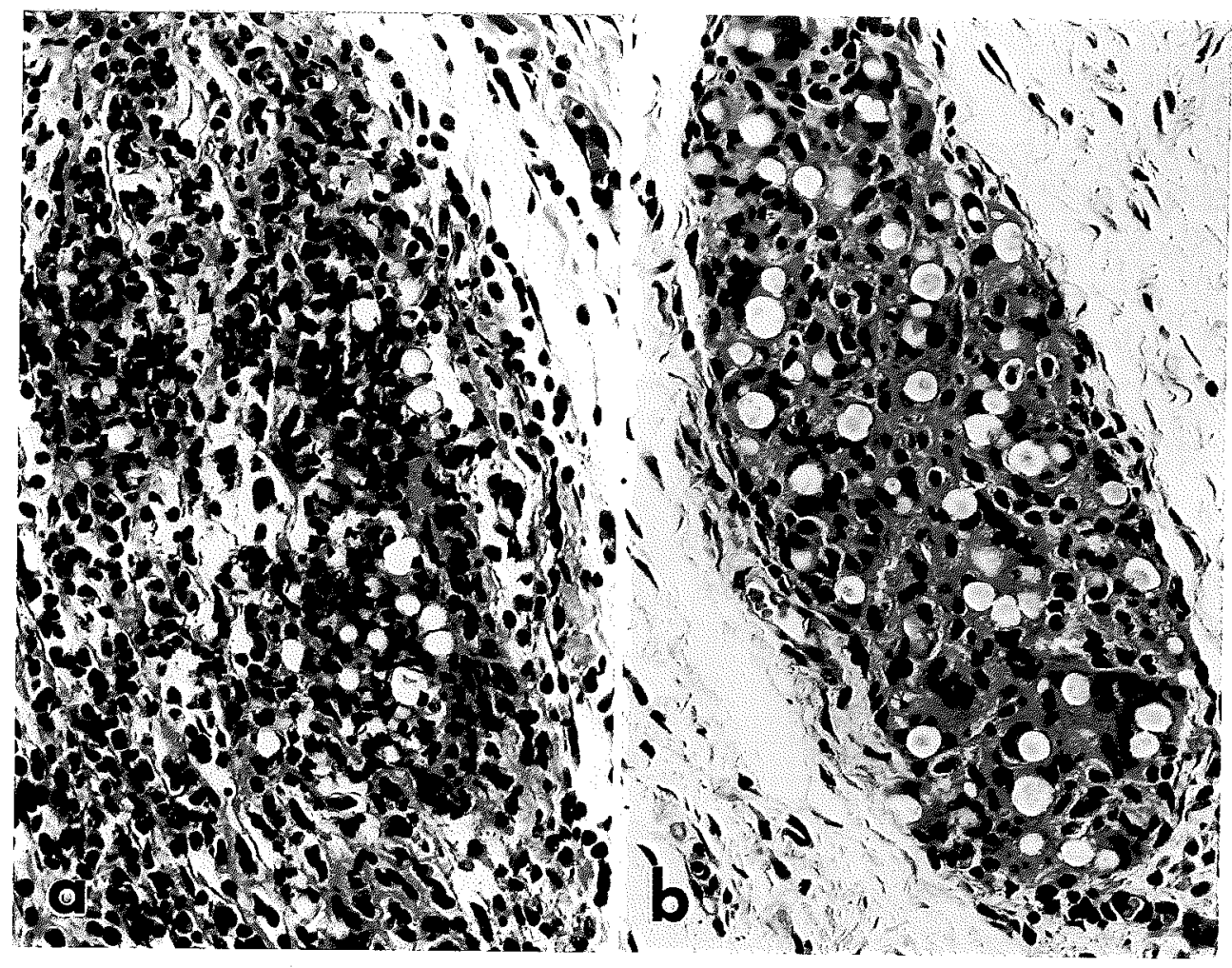

Fig. 7 Light micrographs of tissue responses to larger particles (group-C). a: Massive infiltration of macrophages and a few neutrophils around the particles (1st day). b: The lesion is now compact and spindle-shaped cells are seen in its periphery. Cytoplasmic borders are indistinct (28th day). a, b: $\times 370$. Hematoxylin and eosin staining. Decalcified

microprojections, and hardly any intercellular spaces could be seen. A subplasmalemmal linear density was occasionally observed on complicately intertwined microprojections. The cell surfaces bordering the particles were nearly smooth, and no evidence of destruction or absorption of larger particles by these cells could be found (Fig. 9).

From the 14th to 28th day, macrophages and multinucleated giant cells intimately interdigitated with numerous elongated microprojections; no spaces were present among them.

\section{Ruthenium Red Staining}

Ultrastructurally, macrophages were covered with a fine granular material of varying thickness. This glycocalyx and the numerous coated vesicles of tissue macrophages, immature and mature macrophages and multinucleated giant cells were positive for ruthenium red. In immature macrophages, the ruthenium redpositive layer was very thin. This layer gradually became thicker with the differentiation of the cells. Macrophages and multinucleated giant cells closely and deeply interdigitated, as this layer presevered. When larger particles were completely enclosed by macrophages and multinucleated giant cells, the ruthenium red-positive layer was recognized on the cell surfaces facing the particles (Fig. 10).

\section{Peroxidatic Reaction}

Peroxidatic reaction was tested to investigate the origin of macrophages involved in the lesions. However, no differences in the localizations of the reaction product were observed. In blood monocytes, tissue macrophages, immature and mature macrophages in the lesions, lysosomal granules and mitochondria 


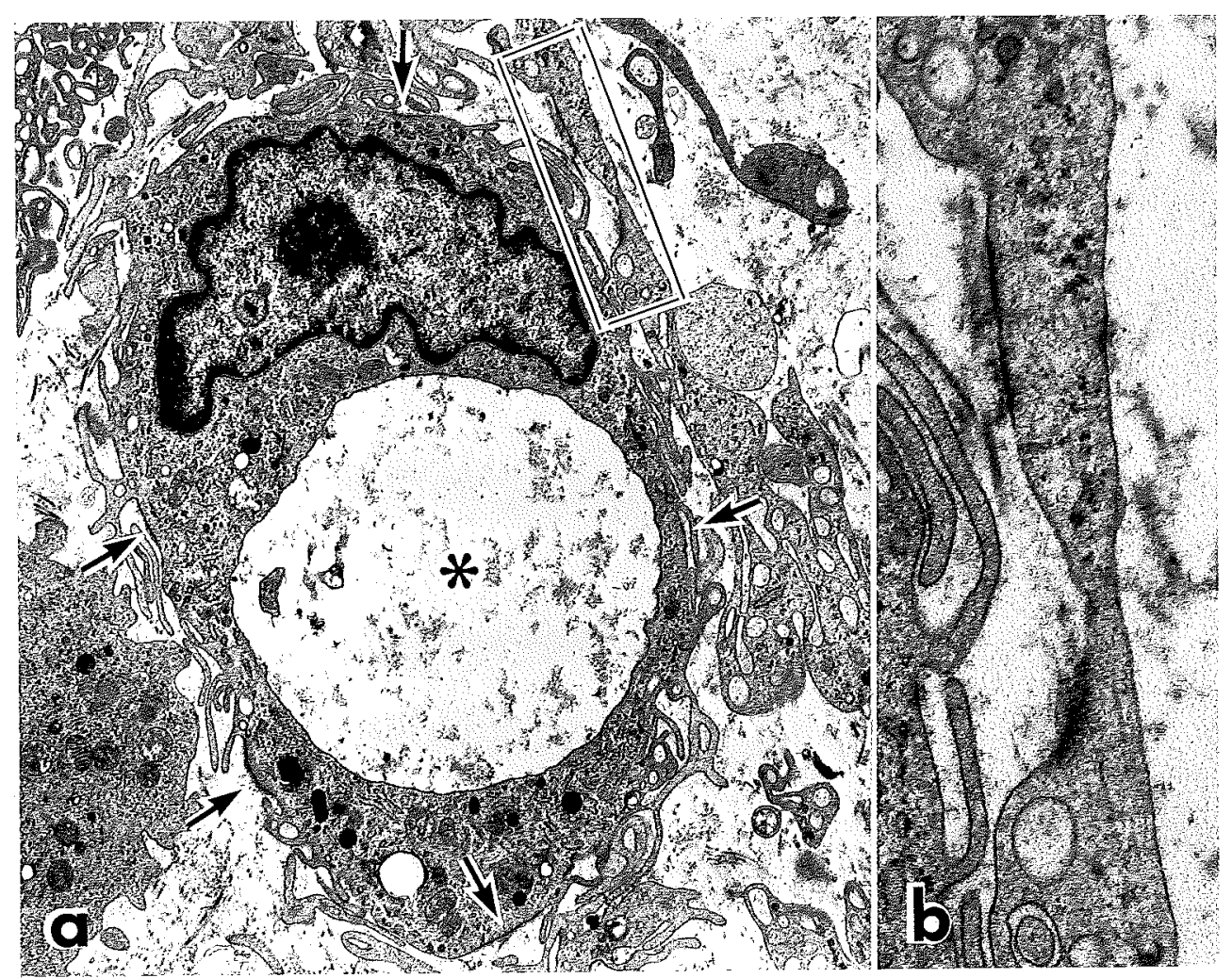

Fig. 8 a: A solitary larger particle (*) is completely surrounded by a macrophage. Subplasmalemmal linear densities (arrows) are seen (group-B, 5th day). $\times 6,700$. b: High magnification of the box, showing subplasmalemmal linear densities. $\times 22,000$. Decalcified

showed positive reactions (Fig. 11). In multinucleated giant cells, no positive reactions could be seen in lysosomal granules.

\section{DISCUSSION}

Previous studies have shown that HAP has excellent biocompatibility in soft tissues, and that larger particles of HAP of hundreds $\mu \mathrm{m}$ in diameter implanted in soft tissue were surrounded by fibrovascular or fibrous tissue occasionally intermingling macrophages and multinucleated giant cells without any tissue damage $(4,7,12)$. The above-mentioned cellular responses were ascribed to physical and mechanical stimuli, and not to chemical ones due to adequate affinity between the implanted materials and surrounding tissues (7).

In the present study, soft tissue responses, especially the reactions of mononuclear phagocytes to fine particles of HAP were examined during a period of 28 days after subcutaneous administrations. The results of the present study were essentially similar to previous reports. These processes are shown in Fig. 12.

Granuloma is defined as a compact collection of mature mononuclear phagocytes with or without an immunological defensive mechanism (1). It is well-known that foreign body granuloma and foreign body reactions are formed as a response to various endogenous or exogenous foreign materials in order to eliminate them. Such small and non-injurious foreign materials such as anthracotic pigments have been known to be phagocytized and disposed of by macrophages. In the case of large, irritant and injurious foreign materials, granulomatous inflammation or encapsulation of materials by a fibrous capsule occurs 


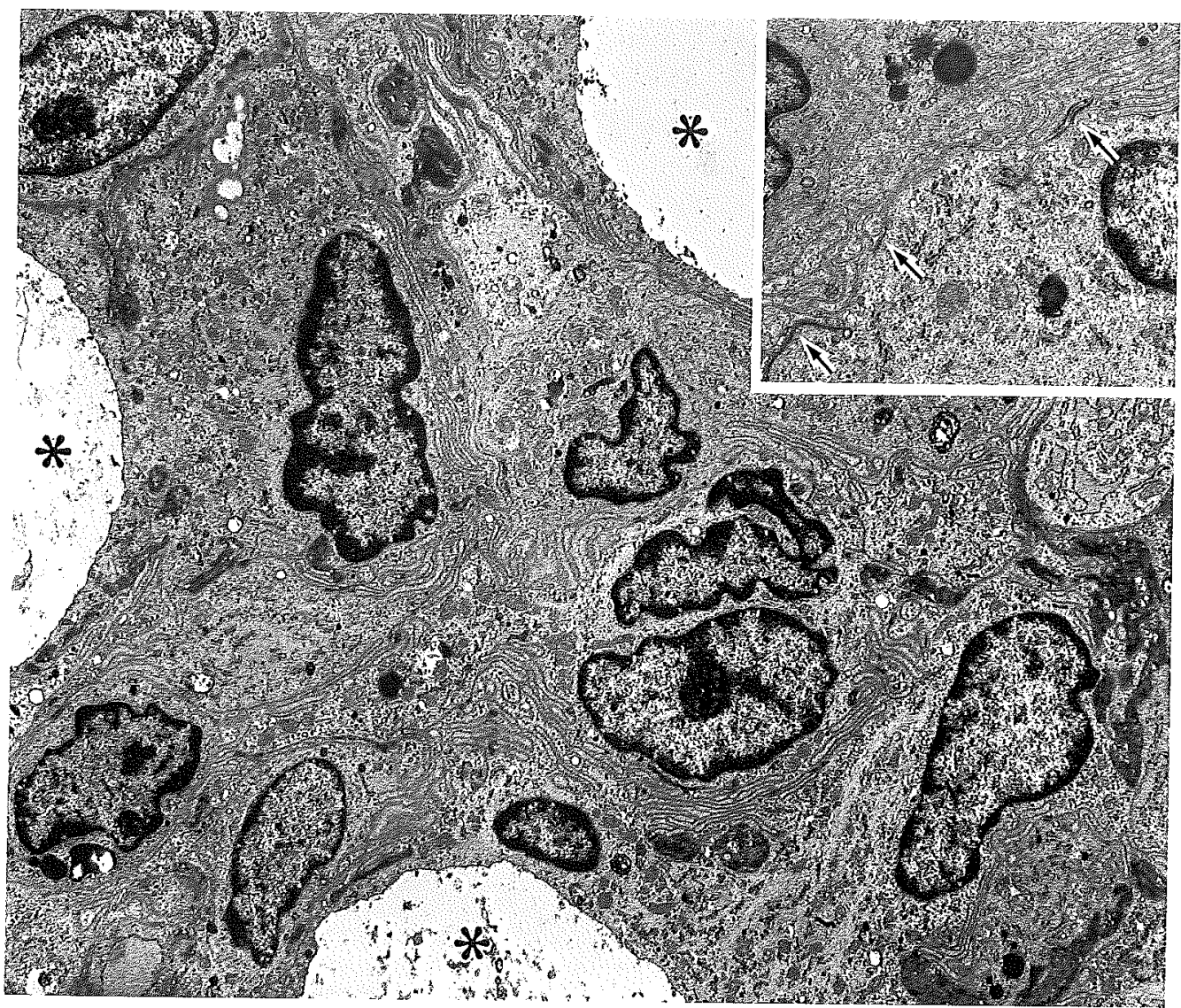

Fig. 9 The tight association of macrophages around larger particles (*) (group-B, 7th day). Note the fine, lamellar interdigitation of the cells. Here intercellular spaces can hardly be recognized. $\times 5,000$. Inset: Subplasmalemmal linear densities (arrows) on complicating microprojections are seen. $\times 8,300$. Decalcified

(21).

Taking into consideration the present observations, it seems reasonable to suggest that the tissue responses to the HAP particles represent a kind of foreign body granuloma which is characterized by the involvement of mononuclear phagocytes and their derivates. Phagocytosis and disposal of smaller particles, localization, and the isolation of larger particles by these cells are the main responses. These responses appear to represent defense mechanisms of the organism for the elimination of foreign materials. No essential differences in the cellular components according to the various sizes of the particles could be found in the present study.

Also in this study, the sequential differentiation of mononuclear phagocytes were recognized as have been reported in various experimentally induced granulomatous lesions $(1,20)$. Morphologically considered, two populations of macrophages were observed in extremely early stages. One was immature macrophages resembling blood monocytes and the other was mature macrophages appearing as tissue macrophages. Infiltrating immature macrophages gradually differentiated into the mature form of macrophages. Two populations of macrophages, preexisting mature macrophages and the mature form of macrophages, could not be distinguished from each other on morphological grounds with the passing of time. On examination of peroxidatic activity, tissue macrophages and cells excluding multinucleated giant cells in the lesions showed positive ac- 


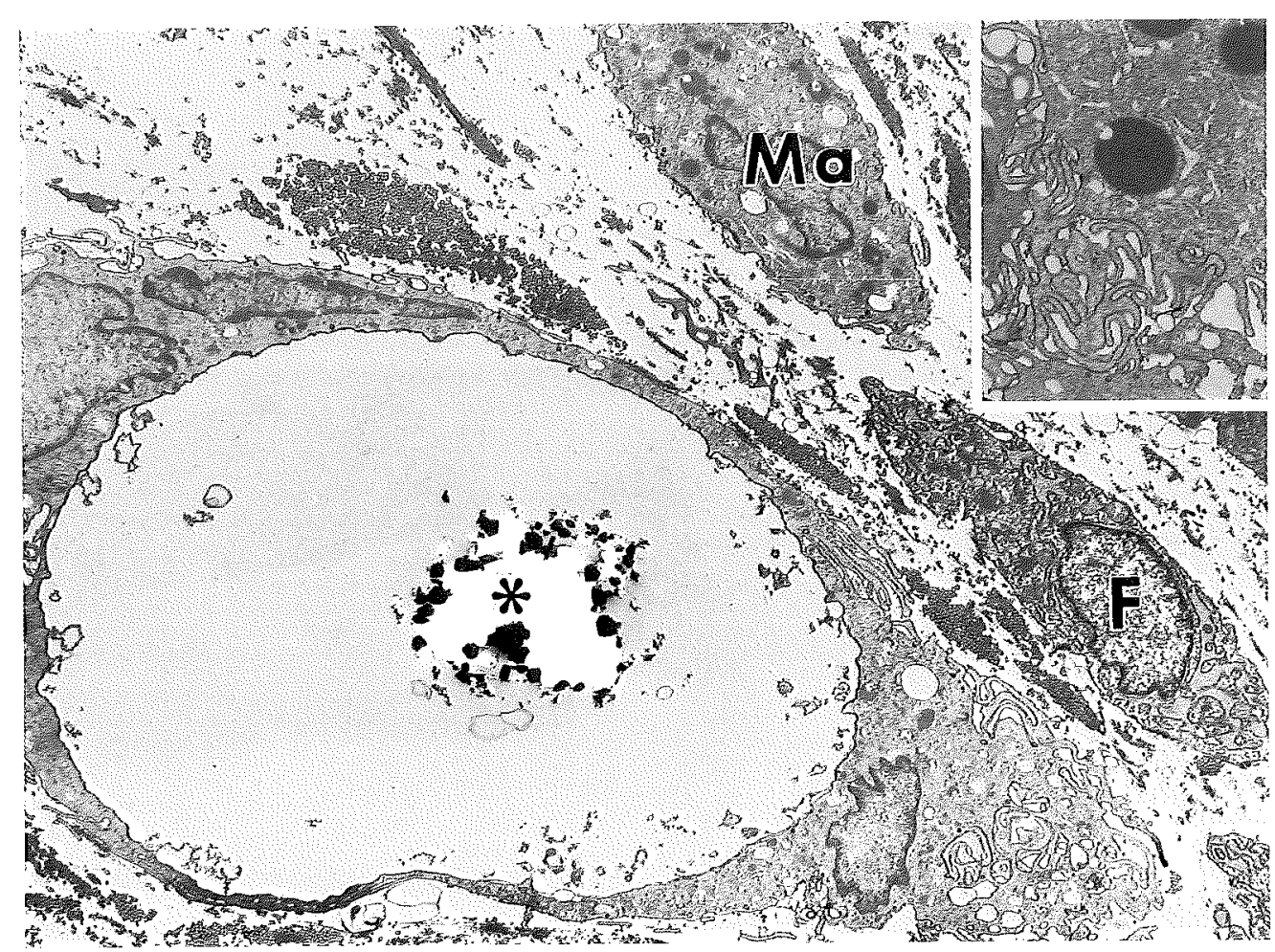

Fig. 10 A macrophage (Ma), multinucleated giant cell and fibroblast (F) are covered with a substance stained with ruthenium red. The cell surface facing a large particle is also covered with the same material (group-B, 5th day). $\times 5,300$. Inset: Intricately interdigitating cell portions are also covered with the same material (group-B, 7 th day). *: small fragments of HAP particle remaining after decalcification. $\times 7,200$. Decalcified

tivities in lysosomal granules and mitochondria. Positive activities were not detected in the rough endoplasmic reticula and nuclear envelope, which have been observed in peritoneal macrophages (3). Additionally, only a few mitotic figures of macrophage populations were observed during the course of the experiments. These findings suggest that rapidly infiltrating immature macrophages at the injection sites are derived from blood monocytes. They then gradually differentiate into mature macrophages and multinucleated giant cells, being composed of the main cellular components of the lesions. However, the participation of pre-existing tissue macrophages can not be discounted.

During these differentiations of the immature macrophages, some characteristic ultrastructural features closely allied to phagocytosis and the encapsulation of HAP particles were recognized. The cell surfaces were covered with glycocalyx, consisting of acid mucopolysaccharides, which has been known to play an important role in the recognition of foreign materials in the process of phagocytosis (2). Papadimitriou indicated that cell surfaces of resident macrophages held a higher negative charge than that of exudate macrophages or multinucleated giant cells (17). It was also reported that a reduction in the charge of the cell membrane is closely related to the process of multinucleation of macrophages, namely proximation, intimate contact between adjacent macrophages, and fusion (18). In the present study, ruthenium red staining was employed not only to visualize the surfaces of cells consisting of the lesions but also to assess morphologically the surface charge of these cells because ruthenium red is a hexavalent anion (10). In immature macro- 


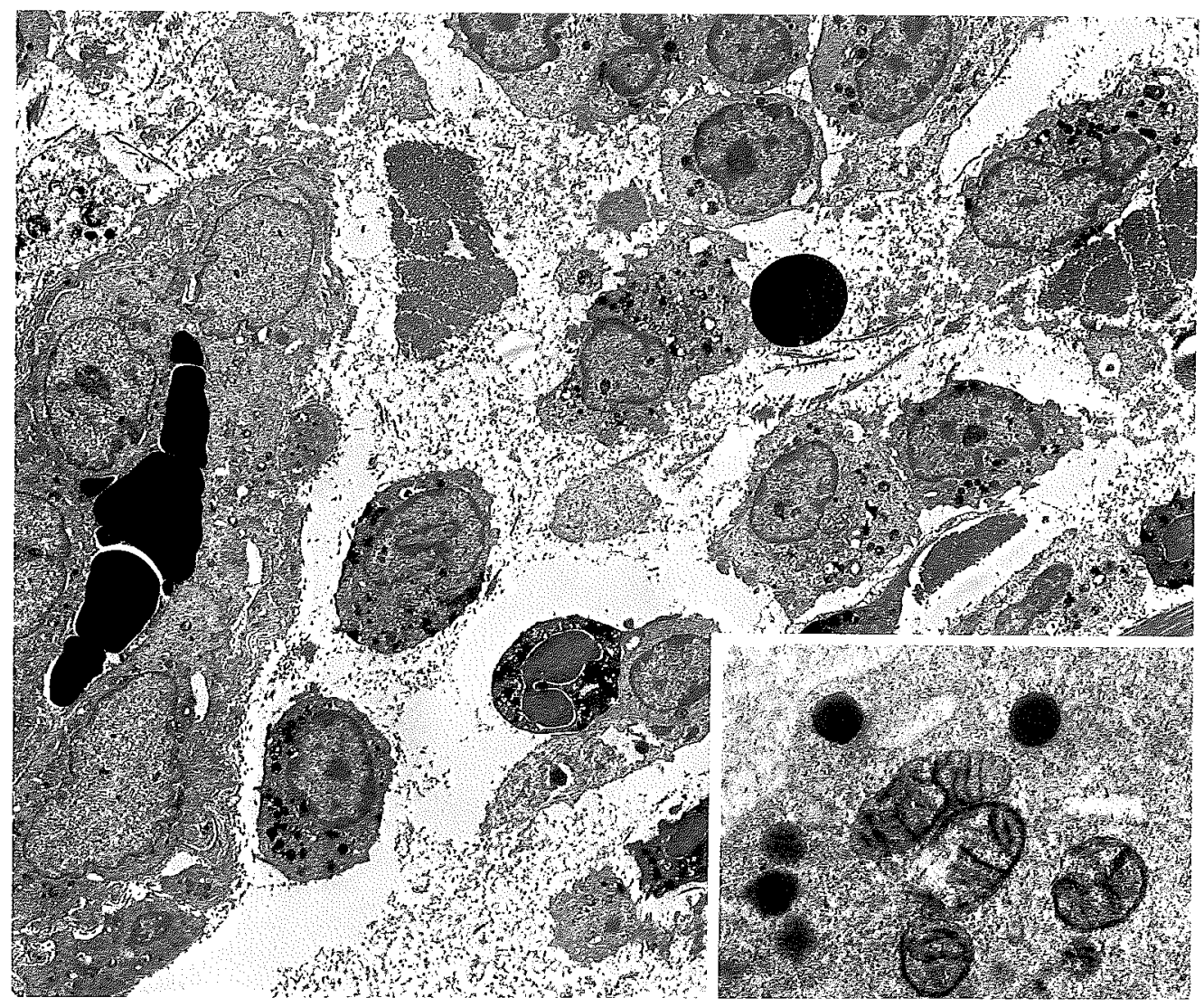

Fig. 11 Peroxidatic activities are demonstrated in lysosomal granules and mitochondria of immature and mature macrophages (group-B, 3rd day). Reaction products appear dark in the micrographs. $\times 2,200$. Inset: In blood monocytes, the reaction products are also observed in lysosomal granules and mitochondria, $\times 20,000$

phages, the ruthenium red positive layer was very thin, becoming thicker as they differentiated. When the lesion maturated, and macrophages and multinucleated giant cells closely approximated and began to interdigitate, this layer could be found though it became gradually thinner. These findings generally corresponded to the change in charge of the cell membrane reported by Papadimitriou. Thus, it is concluded that these increases and decreases in the thickness of glycocalyx are important for the recognition of smaller particles as foreign materials, the intimate contact of macrophages, and the formation of multinucleated giant cells.

Another important ultrastructural finding was the randomly distributed subplasmalemmal linear densities (9) and subplasmalemmal microfilaments (19) located beneath the cell membrane of cells consisting of the lesions. Subplasmalemmal linear densities have been defined as electron dense intracytoplasmic structures, composed of fine granular materials, located immediately subjacent to the cell membrane and found in cells of the mononuclear phagocytic system excluding blood monocytes. This structure has been closely related to the binding of actin filaments to cell membranes and the immobilization of macrophages and multinucleated giant cells in granulomatous lesions (9). Subplasmalemmal microfilaments, a filamentous network composed of 40-50 $\AA$ microfilaments appearing as actin filaments, have been thought to be related to the translocation of the cell membrane in the process of phagocytosis and attachment 


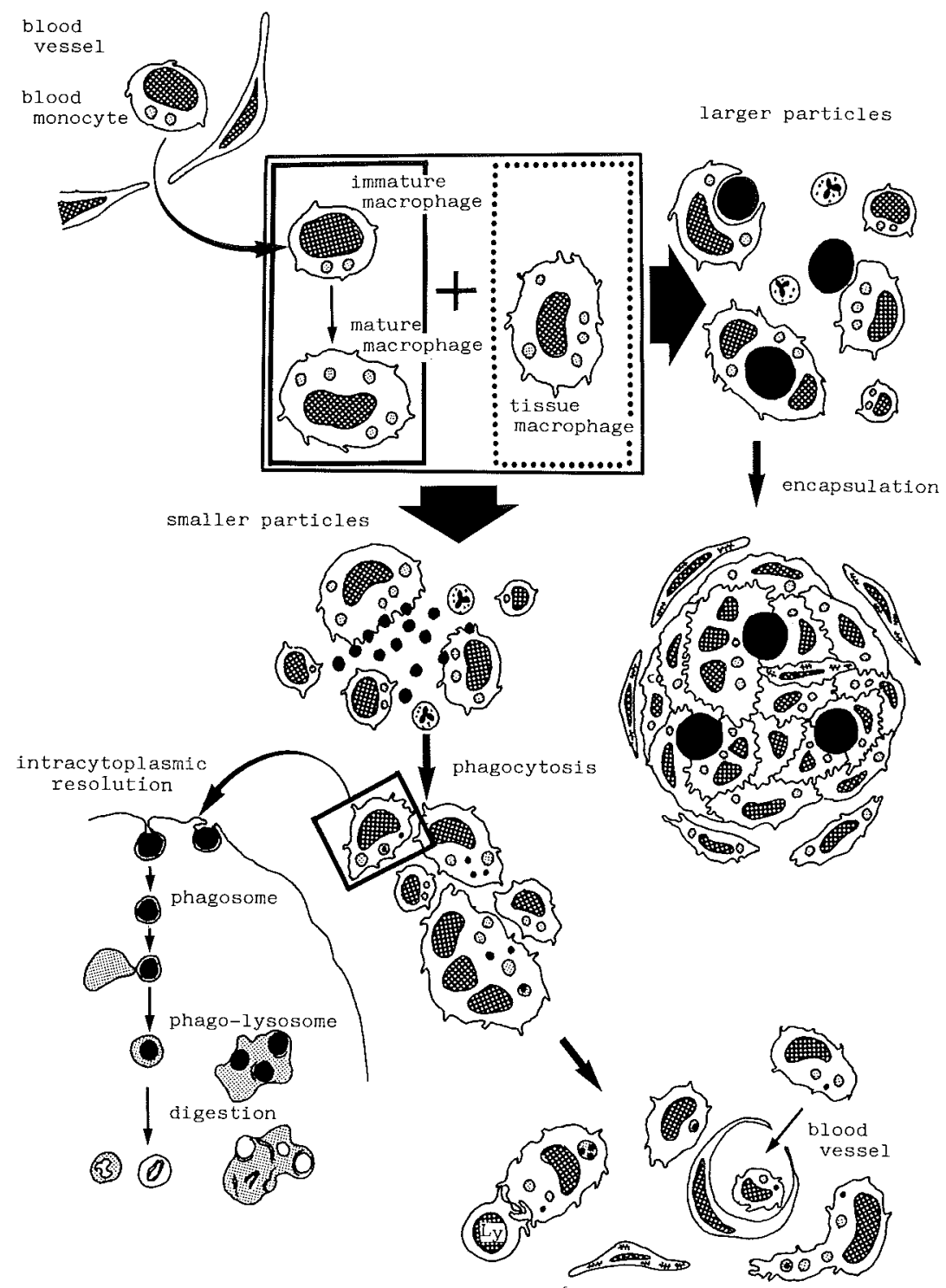

Fig. 12 Schematic diagram showing sequential tissue responses to HAP particles. Ly, lymphocyte; nucleus; , lysosomal granule

to foreign materials (19). In the present study, the existence of subplasmalemmal linear densities in complex intertwined microprojections of compactly aggregated cells around the particles plays a significant role in the localization of HAP particles, although the mechanical interdigitation of tentacular microprojections must also be important. On the other hand, occasionally encountered subplasma- lemmal microfilaments subjacent to cell membranes facing smaller particles are also suited for the capture and ingestion of the particles by invagination and fusion of the cell membrane as contractile elements.

The processes of phagocytosis and the intracytoplasmic resolution of smaller particles were similar to those of other foreign materials reported previously (8). Ohkuma et al. have 
shown that the normal $\mathrm{pH}$ readings of macrophages' lysosomes were 4.7 to 4.8 (16). Taking into account the present morphological fundings and qualities of lysosomes, it is conceivable that some, if not all, phagocytized particles are resolved in a decalcification-like mechanism in the phago-lysosomes of macrophages and multinucleated giant cells. It is also necessary to consider that macrophages laden with smaller particles enter the blood stream and migrate into other organs, such as the spleen, regional lymph nodes and others.

Multinucleated giant cells were observed in all experimental groups. In the case of the administration of smaller particles, they phagocytized numerous small particles. This reaction of multinucleated giant cells to smaller particles is indicative of their property to remove smaller particles efficiently. On the other hand, larger particles were not phagocytized but surrounded by macrophages and multinucleated giant cells. These appearances are thought to be the isolation or absorption of larger particles by these cells. However, morphological evidence of the active absorption of the particles, such as ruffled borders and numerous intracytoplasmic vacuoles observed in osteoclasts which actively absorb bones, can not be found in multinucleated giant cells. Multinucleated giant cells surrounding or facing larger particles are thought to play an essential role in the isolation of the particles.

Lymphocytes and plasma cells were rarely present in the lesions. However, close connections between macrophages laden with smaller particles and lymphocytes by intertwining their elongated cytoplasmic processes were infrequently observed in late stages. It is questionable whether these features may be only accidental phenomena or morphological expressions of the exchange of information on antigen.

The present observations indicate that the soft tissue responses to HAP particles are essentially a non-specific foreign body reaction without any cell or tissue damage. Neither adequate affinity between HAP and surrounding soft tissue nor osteogenesis was seen.

Further observations are needed to clarify the migration of macrophages laden with smaller particles to other organs and bone tis- sue responses to fine particles of HAP such as wear debris which may be brought about in long-term clinical applications of dental implants.

Excellent biocompatibility and adhesive ability to hard and soft tissues are indispensable to biomaterials. Considerations must be given to these qualities in long-term applications.

The author thanks Professor T. Ishiki for his critical reading of the manuscript and helpful comments, and Professor H. Ozawa, the First Department of Oral Anatomy, for his helpful advice. The author is also grateful to the staff of the Department of Oral Pathology. The particles of HAP used in this experiment were supplied by Asahi Optical Company. This study was partly supported by a grant-in-aid for scientific research from the Ministry of Education, Science and Culture, Japan (No. 61480374).

Received for publication 27 December 1987; and in revised form 16 January 1988

\section{REFERENCES}

1. Adams D. O. (1976) The granulomatous inflammatory response. A review. Amer. J. Pathol. 84, 164-191

2. Carr I., Everson G., Rankin A. and RutherFORD J. (1970) The fine structure of the cell coat of the peritoneal macrophage and its role in the recognition of foreign material. $Z$. Zellforsch. 105, 339-349

3. Deams E. Th. and Rhee H. J. van der (1980) Peroxidase and catalase in monocytes, macrophages, epithelioid cells and giant cells of the rat. In Mononuclear Phagocytes Functional Aspects (ed. van FurTh R.) Martinus Nijhoff Publishers, The Hague/Boston/London, pp. 43-60

4. Drobeck H. P., Rothstein S. S., Gumaer K. I., Sherer A. D. and Slighter R. G. (1984) Histologic observation of soft tissue reponses to implanted, multifaceted particles and discs of hydroxylapatite. J. Oral Maxillofac. Surg. 42, 143-149

5. Eggert F. M. and Germain J. P. (1980) Stable acid phosphatase: 1 . Demonstration and distribution. Histochemistry 66, 307-317

6. FAHIMI H. D. (1969) Cytochemical localization of peroxidatic activity of catalase in rat hepatic microbodies (peroxisomes). J. Cell Biol. 43, 275-288

7. Hara Y., Furukawa T., Kishi T., Chen Y.-J., AKamine A. and Aono M. (1983) Application of calcium phosphate ceramics to periodontal therapy 1 . Fundamental studies on hydroxyapa- 
tite. J. Jap. Assoc. Periodontol. 25, 806-816 (in Japanese)

8. KaJiKawa K. (1977) Development and structure of granulation tissue. In Handbook of Inflammatology Vol. 3, Immune Mechanism in Chronic Inflammation (ed. Yasuhira K., TsuruFujI S. and Mizushima Y.) Igaku Shoin, Tokyo, pp. 54-83 (in Japanese)

9. Kawanami O., Ferrans V. J. and Crystal R. G. (1980) Subplasmalemmal linear densities in cells of the mononuclear phagocyte system in lung. Amer. J. Pathol. 100, 131-150

10. LuFT J. H. (1971) Ruthenium red and violet. 1. Chemistry, purification, methods of use for electron microscopy and mechanism of action. Anat. Rec. 171, 347-368

11. Luft J. H. (1971) Ruthenium red and violet. II. Fine structural localization in animal tissues. Anat. Rec. 171, 369-416

12. Misiek D. J., Kent J. N. and Carr R. F. (1984) Soft tissue responses to hydroxyapatite particles of different shapes. J. Oral Maxillofac. Surg. 42, $150-160$

13. Mori M. (1987) Subcutaneous tissue reactions of the rat to synthetic hydroxyapatite ceramic particles.: An Electron Microscopic Study. Niigata Dent. J. 17, 99-115 (in Japanese)

14. Nagahara K. (1987) Osteogenesis in response to tricalcium phosphate (TCP) and hydroxyapatite ceramics (HAP) implanted into bone tissues. Jap. J. Oral Biol. 29, 131-155 (in Japanese)
15. OGIso M. (1983) Formation and calcification of bone tissue on the surface of apatite ceramics. J. Stomatol. Soc. Jap. 50, 1-22 (in Japanese)

16. Ohkuma S. and Poole B. (1978) Fluorescence probe measurement of the intralysosomal $\mathrm{pH}$ in living cells and the perturbation of $\mathrm{pH}$ by various agents. Proc. Natl. Acad. Sci. USA 75, $3327-3331$

17. Papadimitriou J. M. (1982) As assessment of the surface charge of single resident and exudate macrophages and multinucleate giant cells. J. Pathol. 138, 17-24

18. Poste G. (1972) Mechanisms of virus-induced cell fusion. Int. Rev. Cytol. 33, 157-252

19. Reaven E. P. and Axuine S. G. (1973) Subplasmalemmal microfilaments and microtubules in resting and phagocytizing cultivated macrophages. J. Cell Biol. 59, 12-27

20. RheE H. J. VAN DeR, Burgh-De Winter C. P. M. VAN DER and DAEMS W. TH (1979) The differentiation of monocytes into macrophages, epithelioid cells, and multinucleated giant cells in subcutaneous granulomas. 1. Fine structure. Cell Tissue Res. 197, 355-378

21. Sasano N. (1974) Pathological growth. In Textbook of Pathology Vol. 1 (ed. AKAZAKI K.) Nanzando, Tokyo, pp. 191-213 (in Japanese)

22. Tomil Y. (1987) Subcutaneous tissue reactions of the rat to synthetic hydroxyapatite ceramic particles. Niigata Dent. J. 17, 65-82 (in Japanese) 